\title{
Evaluation of a Pharmacist-Managed Amiodarone Monitoring Program
}

\author{
Michele M. Spence, PhD; Jennifer K. Polzin, PharmD; Calvin L. Weisberger, MD; \\ John P. Martin, MD; Jay P. Rho, PharmD; and Giselle H. Willick, PharmD
}

\begin{abstract}
BACKGROUND: Because of the potential for serious adverse effects, patients treated with amiodarone must be carefully screened and routinely monitored for potential liver, thyroid, and pulmonary toxicity. However, laboratory and pulmonary monitoring rates have been found to be substantially lower than recommended in guidelines, including those of the North American Society of Pacing and Electrophysiology (NASPE, 2007).
\end{abstract}

OBJECTIVE: To (a) assess rates of laboratory monitoring of liver, thyroid, and pulmonary function and adverse events in a pharmacist-managed amiodarone monitoring program compared with usual care in an integrated health care system and (b) estimate return on investment (ROI) from this intervention.

METHODS: This retrospective cohort study used clinic and enrollment data to identify those patients in the pharmacist-managed program and usual care who received at least 100 days of amiodarone therapy with the first prescription for amiodarone (index) from June 1, 2007, through May 31 , 2009 (index date). Laboratory test monitoring was recorded at baseline (up to 6 months before the index date), from 1-6 months after the index date, 7-12 months after the index date, and at any time during the year (months 1-12). Alanine aminotransferase (ALT) was evaluated for liver function. Thyroid-stimulating hormone (TSH) and, for patients with abnormal TSH $(<0.4$ micro international units [ulU] per $\mathrm{mL}$ or $>4.0$ ulU per $\mathrm{mL})$, free thyroxine (T4) were evaluated for thyroid function. Rates of pulmonary function testing (PFT) were measured by the diffusion capacity of carbon monoxide tests $\left(D_{L} C O\right)$ and annual chest $x$-rays (CXR); electrocardiograms were not counted. Monitoring rates were compared using Pearson chi-square tests, and logistic regression was used to compare the odds of testing (ALT, TSH, T4, CXR, PFT) between the 2 groups at any time during the year after the index date. Concomitant uses of amiodarone with high-dose statins and of amiodarone with digoxin were compared using Pearson chi-square tests. Hospitalizations and emergency room (ER) visits during the 12-month follow-up period were counted for (a) interstitial lung disease; (b) rhabdomyolysis for patients who received amiodarone with high-dose statins (either lovastatin $>40 \mathrm{mg}$ per day or $>\mathbf{2 0} \mathrm{mg}$ per day of simvastatin or atorvastatin); and (c) for patients with abnormal digoxin, ALT, TSH, or T4 levels, if the hospitalization occurred within 2 days of the abnormal laboratory value.

RESULTS: There were 2,292 patients who received at least 100 days of amiodarone therapy and met the other inclusion criteria, of whom 181 patients $(7.9 \%)$ were in the pharmacist-managed group and 2,111 received usual care. There were $90(49.7 \%)$ new amiodarone users in the pharmacist-managed group and $990(46.9 \%)$ in usual care. The 2 groups had similar demographic characteristics except race, with more whites and fewer African Americans, Asians, and Hispanics in usual care. Laboratory monitoring rates for ALT, TSH, and T4 were significantly higher in the pharmacist-managed group than usual care at the first and second 6 months and at baseline for ALT and TSH but not T4. Baseline CXR rates were significantly higher for the pharmacist-managed group than usual care $\mathbf{( 5 9 . 1 \%}$ vs. $49.3 \% ; P=0.011)$. Few patients in either group received PFT tests at baseline, $6.6 \%$ versus $3.6 \%$ ( $P=0.042)$. After controlling for covariates (age, gender, race, new vs. continuing use, and comorbidities), pharmacistmanaged patients were significantly more likely to have at least 1 ALT test within the year after the index prescription (odds ratio $[0 \mathrm{R}]=3.13,95 \%$ $\mathrm{Cl}=1.12-8.71)$, as well as a TSH test $(\mathrm{OR}=8.13,95 \% \mathrm{Cl}=3.27-20.21)$ and $\mathrm{T} 4(\mathrm{OR}=2.51,95 \% \mathrm{Cl}=1.67-3.75)$. $\mathrm{PFTs}$ were also more likely to be given to these patients ( $O R=5.89,95 \% \mathrm{Cl}=3.86-8.99)$. A higher percentage of patients in the pharmacist-managed group than in usual care were taking a high-dose statin during the 12 -month follow-up period $(47.5 \%$ vs. $36.2 \%$, $P=0.003)$, but of those patients, a greater proportion were switched to another statin $(14.0 \%[\mathrm{n}=12]$ vs. $7.5 \%[\mathrm{n}=57], P=0.037)$ or a lower dose $(9.3 \%[n=8]$ vs. $3.9 \%[n=30], P=0.022)$. Six patients in the usual care group $(0.79 \%$ of patients on high-dose statins) developed rhabdomyolysis, and $5(0.24 \%$ of all patients in usual care) had an admission for interstitial lung disease. The proportions of patients using amiodarone and digoxin concomitantly were similar in the 2 groups $(35.9 \%$ vs. $31.3 \%, P=0.197)$. Among patients with abnormal laboratory results for ALT, TSH, and T4, or digoxin, there were 2 all-cause hospitalizations and 1 ER visit in the pharmacist-managed group and 34 all-cause hospitalizations and 18 ER visits in the usual care group during the follow-up year. Assuming that all hospitalizations and ER visits incurred in the usual care group were avoidable, approximately $\$ 2.14$ could be saved for every dollar spent on the pharmacist-managed amiodarone monitoring program.

CONCLUSIONS: Pharmacist management of patients treated with amiodarone was associated with improved monitoring of recommended laboratory tests and PFTs. Managed care pharmacists can play an instrumental role in implementing case management strategies to improve quality of care for patients treated with amiodarone.

J Manag Care Pharm. 2011;17(7):513-22

Copyright $\odot$ 2011, Academy of Managed Care Pharmacy. All rights reserved.

\section{What is already known about this subject}

- Amiodarone is an effective antiarrhythmic medication but is associated with extensive adverse effects, including potential liver, thyroid, and pulmonary toxicity. Current standards such as those proposed by the North American Society of Pacing and Electrophysiology (NASPE, 2007) recommend thyroid and liver function monitoring at baseline and every 6 months while on amiodarone. Other recommended tests include baseline pulmonary function with repetition as clinically indicated, baseline ophthalmologic tests for patients with significant visual abnormalities, and baseline and yearly chest x-rays (CXRs) and electrocardiograms.

- Previous studies have reported laboratory monitoring rates for amiodarone therapy substantially lower than recommended, with approximately one-half of patients receiving recommended monitoring tests every 6 months. 


\section{What is already known about this subject (continued)}

- Drug-drug interactions with amiodarone include digoxin, warfarin, and high-dose statins (e.g., >40 mg per day of lovastatin or $>20 \mathrm{mg}$ per day of either simvastatin or atorvastatin). Karimi et al. (2010) found that $92(0.52 \%)$ of 17,760 patients at a Veterans Affairs medical center in 2008 who received simvastatin $40 \mathrm{mg}$ or more per day also received amiodarone.

- Recent studies have shown that pharmacist monitoring of amiodarone appears to improve patient adherence to recommended testing protocols. There is limited information on actual harm avoided by reduction in liver, thyroid, or pulmonary toxicity associated with pharmacist monitoring of amiodarone use.

\section{What this study adds}

- Among patients taking a high-dose statin and amiodarone concomitantly, a greater proportion in the pharmacist-managed group were switched to a safer statin $(14.0 \%$ [ $n=12]$ vs. $7.5 \%$ $[\mathrm{n}=57], P=0.037)$ or a lower dose $(9.3 \%[\mathrm{n}=8]$ vs. $3.9 \%[\mathrm{n}=30]$, $P=0.022)$ during the 12 -month study period. In the usual care group, $6(0.28 \%)$ patients who were taking a high-dose statin and amiodarone concomitantly had a hospitalization or emergency room (ER) visit with a primary diagnosis for rhabdomyolysis, compared with no patients in the pharmacist-managed group.

- The proportions of patients with concomitant digoxin and amiodarone use were similar between the 2 groups (35.9\% in the pharmacist-managed group vs. $31.3 \%$ in usual care, $P=0.197$ ). Among those patients, 6 in the pharmacist-managed group and 43 in usual care had serum digoxin levels exceeding 2 nanograms (ng) per $\mathrm{mL}(9.2 \%$ vs. $6.4 \%, P=0.405)$. There were 1 hospitalization and 1 ER visit in the pharmacist-managed group and 25 hospitalizations and 17 ER visits in the usual care group for those patients with elevated digoxin levels.

- Among patients with alanine aminotransferase (ALT) levels of 120 units per liter (u per L) or more, there was 1 hospitalization in the pharmacist-managed group versus 7 hospitalizations and 1 ER visit among usual care patients. For patients with abnormal thyroid-stimulating hormone (TSH $<0.4$ or $>4.0$ micro international units [uIU] per $\mathrm{mL}$ and thyroxine [T4] $<0.81$ or $>1.48 \mathrm{ng}$ per deciliter $[\mathrm{dL}]$, there were 2 hospitalizations in the usual care group only.

- There were 5 hospitalizations for interstitial lung disease among the usual care group only.

- Assuming that all hospitalizations and ER visits incurred in the usual care group were avoidable, we estimated that approximately $\$ 2.14$ was saved for every dollar spent on the program.
A miodarone is an effective medication used for the treatment of life-threatening ventricular arrhythmias. It is also used in the treatment of atrial fibrillation (AF), although the U.S. Food and Drug Administration (FDA) has not approved this indication. ${ }^{1}$ Approximately $16 \%$ of patients with AF have received amiodarone within the first 2 years of treatment. ${ }^{2}$ Amiodarone is distributed to the tissues due to its lipophilic properties and is stored in high concentrations in fat and muscle, as well as in the liver, lungs, and skin. It is eliminated through hepatic metabolism and biliary excretion and has an unusually long half-life, averaging about 58 days. ${ }^{3}$ Amiodarone is associated with extensive adverse effects that include relatively mild effects (e.g., nausea, photosensitivity, skin discoloration) or more serious adverse effects that include toxicity of the liver, thyroid, and lungs.

The reported frequency of amiodarone-induced toxicities is quite variable. A meta-analysis of 6 double-blind, placebocontrolled randomized trials reported the major adverse experiences that were associated with early permanent drug discontinuation. ${ }^{4}$ The study found an absolute net risk (amiodarone rate less placebo rate) of liver toxicity to be $0.6 \%$, with a range from $0.0 \%-8.3 \%$ across the 6 trials. ${ }^{4}$ Hypothyroidism was the most common serious adverse experience, with a net risk of $5.9 \%$ of patients (range $=0.0 \%-15.7 \%$ ). The risk of hyperthyroidism was $0.9 \%$ (range $=0.0 \%-3.6 \%$ ). ${ }^{4}$ Pulmonary toxicity is potentially the most serious adverse effect, and the meta-analysis found a net risk of $1.1 \%$ (range $=0.2 \%-6.3 \%)^{4}$ Although the incidence of this complication has decreased with the use of lower doses of amiodarone, it can occur with any dose. Pulmonary toxicity usually manifests as an acute or subacute pneumonitis, typically with diffuse infiltrates on chest X-ray (CXR) and high-resolution computed tomography. Other, more localized, forms of pulmonary toxicity may occur, including pleural disease, migratory infiltrates, and single or multiple nodules. ${ }^{5,6}$ With early detection, the prognosis is good. Most patients diagnosed promptly respond well to the withdrawal of amiodarone and the administration of corticosteroids, which are usually given for 4 to 12 months. ${ }^{1,7}$

A number of drug-drug interactions with amiodarone may occur, including digoxin and high-dose statins, defined as lovastatin at doses greater than 40 milligrams $(\mathrm{mg}$ ) per day or greater than $20 \mathrm{mg}$ per day of simvastatin or atorvastatin. ${ }^{1,8}$ The mechanism of how amiodarone increases the level of digoxin is not well known. However, evidence exists that there is a decrease in the clearance of digoxin, leading to an increased concentration of the drug. This may be due to the inhibition of P-glycoprotein by amiodarone displacing digoxin from its tissue binding sites. ${ }^{5,6}$ According to the package insert, digoxin serum levels can increase by $70 \%$ after the first dose of amiodarone is administered. It is recommended to monitor and even decrease the dose up to $50 \%$ and consider discontinuing while the patient is on amiodarone. ${ }^{1}$ Amiodarone and its metabolite, 
desethylamiodarone (DEA), are suggested to inhibit various enzymes in the CYP450 enzyme group including CYP3A4. Simvastatin, lovastatin, and atorvastatin are the 3 statins currently on the market that are substrates of the CYP3A4 enyzme. When taken concurrently with amiodarone and at the higher doses, there is a potential for toxicity leading to muscle-related adverse events, such as myopathy and rhabdomyolysis. ${ }^{1,8}$ Because of the potential for serious adverse effects, which can result in significant morbidity or mortality, patients treated with amiodarone must be carefully screened and routinely monitored.

Current standards, such as those proposed by the North American Society of Pacing and Electrophysiology (NASPE, 2007), recommend thyroid and liver function monitoring at baseline and every 6 months while on amiodarone. ${ }^{9}$ Patients are also recommended to have baseline pulmonary function tests (PFTs) performed and repeated as clinically indicated. CXRs are recommended at baseline and then yearly, and electrocardiograms (ECGs) are recommended at baseline and when clinically relevant. However, a number of studies have reported laboratory monitoring rates substantially lower than recommended. A study of 10 managed care organizations in 2003-2004 found that only about one-half of all ambulatory patients on amiodarone received both recommended tests for liver and thyroid function. ${ }^{10}$ Bickford and Spencer (2006) measured adherence to the NASPE guideline for baseline monitoring among 45 patients initiated on amiodarone therapy during a hospital admission at a medical university. The authors found that $82 \%$ received baseline thyroid function tests, and $87 \%$ received baseline liver function tests. ${ }^{11}$ Baseline CXRs occurred in $87 \%$, and PFTs occurred in $24 \%$ of patients. However, only $11 \%$ of these patients received all recommended monitoring tests at baseline and only 20\%-50\% of patients received chronic monitoring at recommended time intervals. ${ }^{11} \mathrm{~A}$ third study performed in a multispecialty group practice in 2008 showed that liver function testing occurred in 53\% of new users of amiodarone and $60 \%$ of ongoing users; thyroid function was assessed in $75 \%$ of new users and in $48 \%$ of ongoing users. ${ }^{12}$ These studies demonstrate that opportunities exist for improved monitoring of amiodarone therapy.

Managed care pharmacists can play an instrumental role in implementing strategies to improve the monitoring of laboratory values for patients on amiodarone. ${ }^{13}$ Recent studies have shown that pharmacist monitoring of amiodarone appears to improve patient adherence to recommended testing protocols. Johnson et al. (2010) evaluated a centralized pharmacy amiodarone monitoring service (AMS) among 917 patients initiated on amiodarone between October 1998 and December 2006. ${ }^{14}$ They found that patients who initiated amiodarone after AMS implementation were more likely to receive alanine aminotransferase (ALT) testing, ECG, and CXR than were patients who initiated amiodarone pre-AMS. There was no significant difference in thyroid-stimulating hormone (TSH) monitoring. In contrast, a study that evaluated the effectiveness of an amiodarone management clinic (AMC) at a Veterans Affairs (VA) medical center found that $66 \%$ of patients in the AMC versus $37 \%$ in the comparison group were compliant with thyroid function monitoring within a year after initiating amiodarone. ${ }^{15}$ They were also more likely to be compliant with liver function monitoring ( $69 \%$ vs. $38 \%$ ).

In a randomized trial conducted by Raebel et al. (2005), a team of clinical pharmacists received computerized alerts about missing laboratory test results for 15 targeted medications and either contacted the patients and reminded them to obtain the test if the physician had ordered it or ordered tests according to the intervention guideline if they had not been previously ordered. ${ }^{16}$ The greatest improvement in rates of monitoring between the intervention group and usual care was for amiodarone, where $79 \%$ of the intervention group received monitoring compared with $51 \%$ in usual care $(P<0.001)$.

Snider et al. (2009) conducted a retrospective review of antiarrhythmic medications clinic outpatients who were monitored by clinical pharmacists from July 2007 to April 2008..$^{17}$ Of a total of 134 patients enrolled in the clinic, 58 were receiving amiodarone and were monitored under protocols based on NASPE guidelines for amiodarone. The authors found that compliance with recommended monitoring tests for amiodarone improved from $20 \%$ before the first pharmacist-managed clinic visit to $100 \%$ after the initial visit. Amiodarone was associated with the highest rate of adverse events (23\% of patient visits); the most common of these adverse events was a clinically significant drug interaction, especially with simvastatin.

Sanoski et al. (1998) evaluated a multidisciplinary amiodarone clinic in an ambulatory setting. ${ }^{18}$ Sixty patients were referred to the clinic by their primary physicians and were followed for an average of 9 months by 4 health care specialists: an electrophysiologist, clinical pharmacist, cardiovascular pharmacy fellow, and an electrophysiology nurse. Before clinic enrollment, patients were being treated with amiodarone by their primary physicians for an average of 16 months. After enrollment in the clinic, laboratory tests, including liver and thyroid function tests and CXRs, were obtained every 6 months, and PFTs were scheduled annually. Before referral to the clinic, only 14 (23\%) patients had laboratory tests performed according the guidelines used in the clinic. After enrollment in the clinic, 54 patients (90\%) received follow-up according to the clinic guidelines $(P<0.001)$. The authors found that previously unrecognized adverse events were detected in 21 (35\%) patients, including 4 patients with pulmonary fibrosis, 7 with hypothyroidism, and 3 with liver enzyme elevation. Six patients (10\%) required discontinuation of amiodarone secondary to toxicity.

A number of these studies have been limited either by a small sample size or the lack of an adequate comparison group. 
None reported the cost-effectiveness of the interventions.

Our study sought to evaluate a pharmacist-managed amiodarone program compared with usual care in an integrated health care system. We compared liver, thyroid, and pulmonary function monitoring rates between the 2 groups. The proportions of patients on concomitant use of amiodarone and interacting high-dose statins (defined as lovastatin $>40 \mathrm{mg}$ per day or simvastatin or atorvastatin $>20 \mathrm{mg}$ per day) were compared as well as how many were switched to an equipotent safer statin or a lower dose of the current statin if low-density lipoprotein cholesterol (LDL-C) was controlled. Among those patients, we report hospitalizations and emergency room (ER) visits with a principal diagnosis of rhabdomyolysis. We measured all-cause hospitalization and ER use for patients with toxic digoxin levels exceeding 2 nanograms per milliliter (ng per $\mathrm{mL}$ ) and for patients with abnormal liver or thyroid function tests, as well as hospitalizations and ER visits with a principal diagnosis of interstitial lung disease. Finally, we estimated the return on investment (ROI) of the pharmacist-managed program.

\section{Description of Pharmacist-Managed Amiodarone Program}

The pharmacist-managed amiodarone program was composed of a clinical pharmacist working under an organization-specific protocol authorized by the physicians at 2 urban Kaiser Permanente Southern California (KPSC) medical centers. (A description of the KPSC monitoring guideline is presented in Table 1.) The guideline recommended monitoring of liver (ALT) and thyroid function (TSH and thyroxine [T4] if TSH was $<0.4$ or $>4.0$ micro international units per milliliter [uIU per $\mathrm{mL}]$ ) at baseline and every 6 months. It also recommended baseline and annual CXRs, PFTs (including diffusion capacity of carbon monoxide $\left[\mathrm{D}_{\mathrm{L}} \mathrm{CO}\right]$ ), and ECGs. The patients were not referred to the service but were identified through an electronic medical record (EMR) tool on a monthly basis. Patients on chronic amiodarone therapy were automatically enrolled into the program once the physician approved. Enrollment into the service began on June 1, 2007. A database system tool that pulled data from the EMR tracked adherence to laboratory tests and procedures that were ordered by the pharmacist, and letters were generated by the pharmacist and sent to patients to let them know to come in to complete the tests. If the patient was overdue for a test, the pharmacist would send a reminder by letter or telephone call. The tool also identified patients who were concurrently receiving an interacting statin or digoxin. The pharmacist worked directly with the physician to (a) switch patients to a safer equipotent statin (rosuvastatin or pravastatin, both nonformulary drugs) or a lower dose of the current statin if the LDL-C was controlled and (b) order digoxin serum levels.

The most important role the pharmacist served consisted of actions taken if the results from the ALT, TSH/T4, PFT/CXR, ECG, or digoxin serum tests were abnormal. The pharmacist

\section{TABLE 1 Kaiser Permanente Southern California Guideline for Recommended Testing in Patients Receiving Amiodarone ${ }^{a}$}

\begin{tabular}{|c|c|}
\hline Type of Test & Time When Test Is Performed \\
\hline Liver function tests (ALT) & Baseline and every 6 months \\
\hline $\begin{array}{l}\text { Thyroid function tests (TSH and T4 } \\
\text { if TSH }<0.4 \text { or }>4.0 \text { uIU per mL) }\end{array}$ & Baseline and every 6 months \\
\hline Chest x-ray film & Baseline and every 12 months \\
\hline $\begin{array}{l}\text { Pulmonary function tests } \\
\text { (including } D_{L} C O \text { ) }\end{array}$ & Baseline and every 12 months \\
\hline Electrocardiogram & Baseline and every 12 months \\
\hline \multicolumn{2}{|c|}{$\begin{array}{l}\text { aIf clinical circumstances warrant, more frequent follow-up may be necessary. } \\
\text { ALT = alanine aminotransferase; } D_{L} C O=\text { diffusion capacity of carbon monoxide; } \\
T 4=\text { thyroxine; } T S H=\text { thyroid-stimulating hormone; } U I U \text { per } m L=\text { micro interna- } \\
\text { tional units per milliliter. }\end{array}$} \\
\hline
\end{tabular}

would initiate or adjust all the thyroid hormone medication while consulting with the primary care physician. The pharmacist would decrease the digoxin dose by $50 \%$ or discontinue digoxin in consultation with the cardiologist. ${ }^{1}$ All situations involved consulting with the cardiologist to determine if amiodarone should be discontinued versus adjusting the digoxin or thyroid medication. For abnormal ALT results, the pharmacist would work with the cardiologist to discontinue the amiodarone. If the ECG was abnormal, the pharmacist would work with the cardiologist in adjusting the dose or discontinuing the amiodarone. For abnormal CXRs and/or PFTs, the pharmacist worked with both the cardiologist and pulmonologist to make a treatment plan and discontinue amiodarone. Pharmacists in this program did not monitor international normalized ratio (INR) values for patients on warfarin and amiodarone.

\section{Methods}

The setting for this study included the 10 KPSC centers that provide health care to approximately 3 million members. Our study used a retrospective cohort design based on KPSC clinical and administrative databases. A list of medical record numbers for patients seen in the pharmacist-managed program at 2 of the medical centers identified the pharmacist-managed group. The usual care group was identified using prescription fill history and enrollment data from the other 8 medical centers without pharmacist-managed amiodarone monitoring. All patients in the study received at least 1 prescription for amiodarone from June 1, 2007, through May 31, 2009. The index date was defined as the date of the first prescription for amiodarone (or renewal prescription for continuing patients) from June 1 , 2007, through May 31, 2009. Patients were followed for 1 year after the index date.

To be included in the study, patients had to be aged at least 18 years and enrolled with a pharmacy benefit for at least 1 year before and 1 year after the index date. Patients were excluded if they received less than 100 days of amiodarone, were pregnant, 


\section{FIGURE 1 Cohort Selection Flowchart}

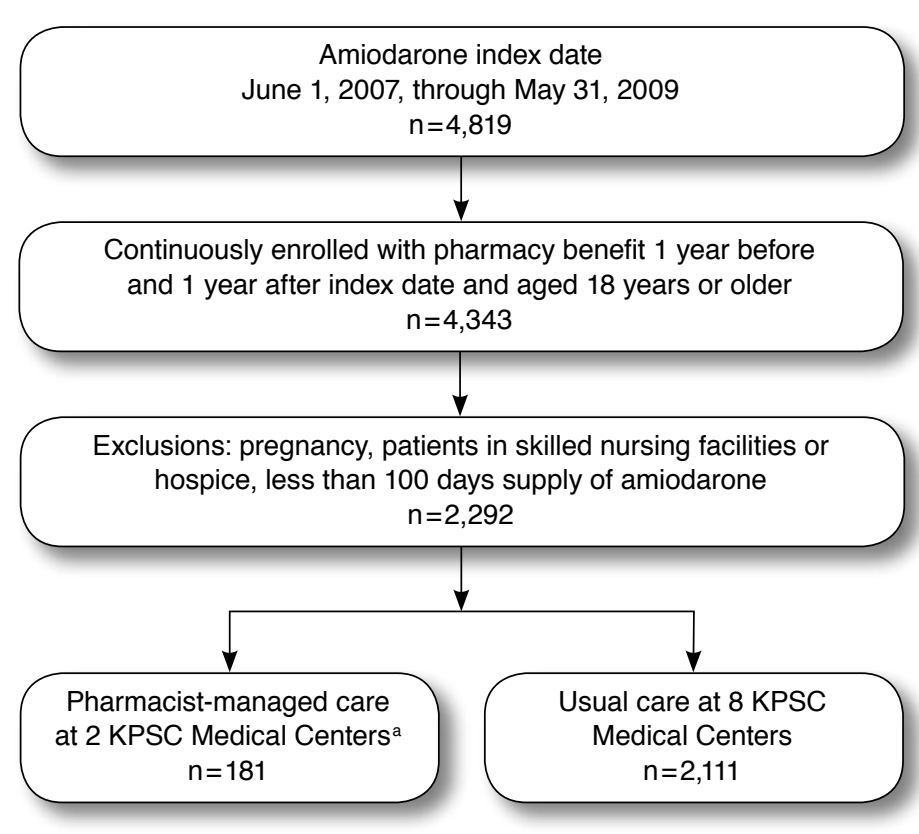

aEnrollment required physician approval; $100 \%$ of patients received physician approval.

KPSC = Kaiser Permanente Southern California

in skilled nursing facilities, or hospice care. Patients were not randomly assigned to the pharmacist-managed group or usual care; rather, all patients on amiodarone who were members at the 2 KPSC medical centers were enrolled into the pharmacistmanaged group with physician approval and were compared with all other patients on amiodarone who were members at 8 other KPSC medical centers (Figure 1).

We compared laboratory test monitoring rates between the pharmacist-managed and usual care groups at baseline, during the first 6 months (months 1-6), during the second 6 months (months 7-12), and at any time during the year (months 1-12) after the index date. Because clinicians might rely on laboratory test results up to 6 months before initiation of a drug, we defined baseline as 180 days before the index date. Laboratory tests were identified using Current Procedural Terminology, Fourth Edition (CPT-4) codes and included codes for both single tests and for test panels that may have included 1 or more tests for liver or thyroid function. ALT (CPT-4 code 84460) was evaluated for liver function, and TSH (CPT-4 code 84443) and T4 (CPT-4 code 84439) were evaluated for thyroid function. Laboratory tests that may have been part of a test panel were captured using internal KPSC descriptions of the specific tests, for example, general health panel CPT-4 code 80050 with a description from the laboratory database of "Thyroid Stimulating Hormone." Rates of PFT were measured by $\mathrm{D}_{\mathrm{L}} \mathrm{CO}$
(CPT-4 code 94720) and annual CXRs (CPT-4 code 7102x). ECGs and ophthalmologic exams were not included because of limited data.

As a summary measure, we compared monitoring of each test (ALT, TSH, T4, CXR, PFT) at any time during the year after the index date, adjusting for the following covariates: age, gender, race, new versus continuing use, and comorbidities. Hospitalizations for interstitial lung disease 1 year after the index date were identified using diagnosis-related group (DRG) codes 092 and 093, and ER visits for interstitial lung disease were identified using International Classification of Diseases, Ninth Revision, Clinical Modification (ICD-9-CM) principal diagnosis codes 515, 516.3, and 516.8. Hospitalizations and ER visits for rhabdomyolysis were identified using ICD-9-CM principal diagnosis code 728.88 .

Concomitant uses of amiodarone with digoxin and lovastatin greater than $40 \mathrm{mg}$ per day or simvastatin or atorvastatin greater than $20 \mathrm{mg}$ per day were identified using a previously validated method. ${ }^{19}$ This method required the days supply of amiodarone with the conflicting drug to overlap by 10 or more days. Concomitant use was counted only once for a given patient, regardless of the number of fills for either drug. If patients were on more than 1 conflicting drug, they were counted for each of the conflicting drugs. The percentages of patients who were on either of the high-dose statins and, of those, the percentages who were switched to either another statin (rosuvastatin or pravastatin) or a lower dose ( $\leq 40 \mathrm{mg}$ of lovastatin, $\leq 20 \mathrm{mg}$ of simvastatin or atorvastatin), or discontinued use were also evaluated during the year after the index date. Abnormal laboratory results were defined as follows: ALT of 120 units per liter (u per L) or more, TSH of less than 0.4 uIU per $\mathrm{mL}$, TSH of more than $4.0 \mathrm{uIU}$ per $\mathrm{mL}$, T4 of less than 0.81 ng per $\mathrm{dL}, \mathrm{T} 4$ of more than $1.48 \mathrm{ng}$ per $\mathrm{dL}$, or digoxin levels more than $2.0 \mathrm{ng}$ per $\mathrm{mL}$. Any all-cause hospitalization or ER visit among those patients with abnormal laboratory levels was also recorded. The abnormal laboratory values had to occur within 2 days prior to the hospitalization or ER visit.

Estimated cost avoidance was based on a rate of $\$ 1,545$ per day for hospitalizations and $\$ 451$ for ER visits, the average rate for our closed health care system. The pharmacist spent approximately 10 hours per month on this service at an estimated health system cost of $\$ 86.88$ per hour (salary plus benefits). Total cost avoidance and total expense of the service were used to estimate an ROI for the pharmacist-managed program.

Comparisons between the pharmacist-managed program and usual care were made using Pearson chi-square tests for differences in percentages and t-tests for differences in means. Multivariate logistic regression was used to compare monitoring in the year after the index date between the 2 groups. Odds ratios (ORs) were reported, adjusting for age, gender, race, new versus ongoing use, and Diagnostic Cost Groups (DxCG) comorbidity score. These covariates were used because provider 


\begin{tabular}{|c|c|c|c|}
\hline \multirow[b]{3}{*}{ Variable } & \multicolumn{3}{|c|}{$\begin{array}{l}\text { haracteristics of Patients Enrolled } \\
\text { Pharmacist-Managed Amiodarone } \\
\text { onitoring Program Versus Usual Care }\end{array}$} \\
\hline & $\begin{array}{l}\text { Pharmacist-Managed } \\
\qquad \mathrm{n}=181\end{array}$ & $\begin{array}{c}\text { Usual Care } \\
n=2,111\end{array}$ & \\
\hline & & & Value $^{\mathrm{a}}$ \\
\hline Age (years) mean [SD] & $71.4 \quad[12.5]$ & $70.0 \quad[11.2]$ & 0.130 \\
\hline Male & $(72.4)$ & $1,449 \quad(68.6)$ & 0.297 \\
\hline \multicolumn{4}{|l|}{ Race } \\
\hline White & $(50.3)$ & $1,450 \quad(68.7)$ & $<0.001$ \\
\hline African American & $(26.0)$ & $127 \quad(6.0)$ & $<0.001$ \\
\hline Hispanic & $(7.2)$ & (3.5) & 0.011 \\
\hline Asian & $(12.2)$ & $(5.0)$ & $<0.001$ \\
\hline Other & $(2.2)$ & $88 \quad(4.2)$ & 0.198 \\
\hline Unknown & $(2.2)$ & $268 \quad(12.7)$ & $<0.001$ \\
\hline New amiodarone users & $\begin{array}{ll}90 & (49.7) \\
\end{array}$ & $990 \quad(46.9)$ & 0.465 \\
\hline $\begin{array}{l}\text { Amiodarone days } \\
\text { supplyb mean [SD] }\end{array}$ & $316.6 \quad[108.2]$ & 309.5 [122.9] & 0.403 \\
\hline $\begin{array}{l}\text { DxCG comorbidity } \\
\text { score mean [SD] } \\
\end{array}$ & {$[2.5]$} & $2.6 \quad[2.4]$ & 0.815 \\
\hline \multicolumn{4}{|c|}{$\begin{array}{l}\text { aPearson chi-square statistical test for percentages and t-tests for means } \\
\text { bDays supply for the 12-month follow-up period after the index date. } \\
\text { DxCG=Diagnostic Cost Group; SD=standard deviation. }\end{array}$} \\
\hline
\end{tabular}

adherence to monitoring may be affected by the extent of patient comorbidities (i.e., sicker patients or new users may be tested more often), or, alternatively, patients' ability or willingness to come in for tests may be affected by demographic characteristics or comorbidities. New use was defined as an initial dispensing of amiodarone during the study period (the index date), with no dispensing in the 6 months preceding the index date. We used the DxCG score as a measure of comorbidity because this score is similar to the risk-adjustment method used by the Centers for Medicare \& Medicaid Services and includes patient encounter-based indicators for a variety of chronic conditions, including coronary heart disease, heart failure, diabetes, and hypertension. ${ }^{20}$

A $P$ value of 0.05 determined statistical significance. All analyses were conducted using SAS version 9.2 (SAS Institute Inc., Cary, NC). The Institutional Review Board of KPSC approved this study.

\section{Results}

A total of 2,292 patients met the study criteria (Figure 1). There were 181 patients (7.9\%) in the pharmacist-managed amiodarone monitoring group and 2,111 in usual care. Demographic characteristics were similar between the 2 groups, with the exception of race (Table 2). Significantly higher percentages of African Americans, Hispanics, and Asians were treated in the pharmacist-managed group than usual care. A significantly higher percentage of patients in usual care was classified as unknown racial origin. Approximately one-half of the patients in each group were new users of amiodarone. The average days
TABLE 3 Comparison of Laboratory and Pulmonary Monitoring Among Patients Enrolled in a Pharmacist-Managed Amiodarone Program Versus Usual Care

\begin{tabular}{|c|c|c|c|c|c|}
\hline \multirow[b]{2}{*}{ Variable } & \multicolumn{2}{|c|}{$\begin{array}{c}\text { Pharmacist-Managed } \\
n=181\end{array}$} & \multicolumn{2}{|c|}{$\begin{array}{l}\text { Usual Care } \\
\mathrm{n}=2,111\end{array}$} & \multirow{2}{*}{$\begin{array}{c}P \\
\text { Value }\end{array}$} \\
\hline & & (\%) & & & \\
\hline ALT: Baseline ${ }^{b}$ & 152 & $(84.0)$ & 1,611 & $(76.3)$ & 0.019 \\
\hline Months 1-6 & 153 & $(84.5)$ & 1,472 & $(69.7)$ & $<0.001$ \\
\hline Months 7-12 & 137 & $(75.7)$ & 1,299 & $(61.5)$ & $<0.001$ \\
\hline Months 1-12 & 172 & (95.0) & 1,839 & $(87.1)$ & 0.002 \\
\hline TSH: Baseline & 127 & $(70.2)$ & 1,324 & $(62.7)$ & 0.046 \\
\hline Months 1-6 & 148 & (81.8) & 1,060 & $(50.2)$ & $<0.001$ \\
\hline Months 7-12 & 140 & $(77.3)$ & 989 & $(46.8)$ & $<0.001$ \\
\hline Months 1-12 & 170 & (93.9) & 1,483 & (70.3) & $<0.001$ \\
\hline T4: Baseline & 44 & $(24.3)$ & 525 & $(24.9)$ & 0.867 \\
\hline Months 1-6 & 75 & $(41.4)$ & 495 & $(23.4)$ & $<0.001$ \\
\hline Months 7-12 & 75 & $(41.4)$ & 454 & $(21.5)$ & $<0.001$ \\
\hline Months 1-12 & 101 & $(55.8)$ & 717 & $(34.0)$ & $<0.001$ \\
\hline CXR: Baseline & 107 & (59.1) & 1,041 & (49.3) & 0.011 \\
\hline Months 1-6 & 78 & $(43.1)$ & 740 & (35.1) & 0.030 \\
\hline Months 7-12 & 59 & $(32.6)$ & 625 & $(29.6)$ & 0.399 \\
\hline Months 1-12 & 103 & $(56.9)$ & 1,056 & $(50.0)$ & 0.076 \\
\hline PFT: Baseline & 12 & (6.6) & 76 & (3.6) & 0.042 \\
\hline Months 1-6 & 61 & (33.7) & 187 & (8.9) & $<0.001$ \\
\hline Months 7-12 & 46 & $(25.4)$ & 140 & $(6.6)$ & $<0.001$ \\
\hline Months 1-12 & 94 & (51.9) & 295 & $(14.0)$ & $<0.001$ \\
\hline
\end{tabular}

aP values from Pearson chi-square statistical test.

${ }^{b}$ Baseline defined as up to 180 days before index date.

ALT = alanine aminotransferase; $C X R=$ chest $x$-ray; PFT = pulmonary function test; T4 =thyroxine; $\mathrm{TSH}=$ thyroid-stimulating hormone.

supply of amiodarone in the 12-month period after the index date was similar in the 2 groups as were the DxCG comorbidity scores.

\section{Laboratory Monitoring Rates}

Laboratory monitoring rates for ALT were significantly higher for the pharmacist-managed group than usual care (Table 3). At baseline, $84.0 \%$ of the patients in the pharmacist-managed group had been tested, compared with $76.3 \%$ in usual care $(P=0.019)$. During the first 6 months after index, $84.5 \%$ of the patients in the pharmacist-managed group had been tested, compared with $69.7 \%$ in usual care $(P<0.001)$. ALT monitoring rates continued to be significantly higher for the pharmacist-managed group in months $7-12$ (75.7\% vs. 61.5\%, respectively, $P<0.001$ ) and overall for months $1-12$ (95.0\% vs. $87.1 \%, P=0.002$ ) following the index date. Monitoring rates for TSH were also significantly higher in the pharmacist-managed group than in usual care at baseline (70.2\% vs. $62.7 \%$, respectively, $P=0.046$ ), during months $1-6$ (81.8\% vs. $50.2 \%$, $P<0.001)$, months $7-12$ (77.3\% vs. $46.8 \%, P<0.001)$, and during the entire year of follow-up (93.9\% vs. $70.3 \%, P<0.001)$. There was no significant difference in rates of $\mathrm{T} 4$ monitoring 


\begin{tabular}{|c|c|c|c|}
\hline $1 \mathrm{AB}$ & \multicolumn{3}{|c|}{$\begin{array}{l}\text { Logistic Regression Analyses } \\
\text { of Laboratory and Pulmonary } \\
\text { Monitoring During the Year } \\
\text { of Follow-up Among Patients } \\
\text { Enrolled in a Pharmacist-Managed } \\
\text { Amiodarone Monitoring Program }\end{array}$} \\
\hline Monitoring Test & $\begin{array}{l}\text { Odds Ratiob } \\
(95 \% \text { CI })\end{array}$ & $P$ Valuec & C-Statistic \\
\hline ALT & $3.13 \quad(1.12-8.71)$ & 0.029 & 0.649 \\
\hline TSH & $8.13 \quad(3.27-20.21)$ & $<0.001$ & 0.610 \\
\hline T4 & $2.51 \quad(1.67-3.75)$ & $<0.001$ & 0.591 \\
\hline CXR & $(0.76-1.79)$ & 0.469 & 0.742 \\
\hline PFT & $5.89 \quad(3.86-8.99)$ & $<0.001$ & 0.706 \\
\hline \multicolumn{4}{|c|}{$\begin{array}{l}\text { aAll equations were adjusted by age, gender, race, new versus continuing user, anc } \\
\text { DxCG comorbidity score. } \\
\text { bOdds ratios compare pharmacist-managed care with usual care. } \\
\text { 'Wald chi-square statistical test. } \\
\text { ALT = alanine aminotransferase; } C I=\text { confidence interval; CXR = chest } x \text {-ray; } \\
\text { DXCG = Diagnostic Cost Group; PFT= pulmonary function test; T4 = thyroxine; } \\
\text { TSH = thyroid-stimulating hormone. }\end{array}$} \\
\hline
\end{tabular}

at baseline, but they were significantly higher than usual care during months $1-6$ (41.4\% vs. $23.4 \%$, respectively, $P<0.001$ ), months $7-12$ ( $41.4 \%$ vs. $21.5 \%, P<0.001$ ), and months $1-12$ (55.8\% vs. $34.0 \%, P<0.001)$.

CXR rates were significantly higher for the pharmacistmanaged group than in usual care at baseline $(59.1 \%$ vs. $49.3 \%$, respectively, $P=0.011$ ) and during the first 6 months ( $43.1 \%$ vs. $35.1 \%, P=0.030$ ). There were no significant differences in CXR rates in months $7-12$ or during months $1-12$. For PFT, monitoring rates were slightly higher in the pharmacist-managed program at baseline $(6.6 \%$ vs. $3.6 \%, P=0.042)$ and substantially higher during months $1-6(33.7 \%$ vs. $8.9 \%, P<0.001)$. A significant difference between the 2 groups remained during the last 6 months of follow-up $(25.4 \%$ vs. $6.6 \%, P<0.001)$ and overall for months $1-12$ (51.9\% vs. $14.0 \%, P<0.001$ ).

After controlling for relevant covariates in logistic regression analyses, the odds of receiving at least 1 ALT test within the year after the index prescription were 3 times as high for pharmacist-managed patients compared with usual care patients $(\mathrm{OR}=3.13,95 \%$ confidence interval $[\mathrm{CI}]=1.12-8.71$; Table 4). Odds were 8 times as high for getting a TSH test $(\mathrm{OR}=8.13$, 95\% CI $=3.27-20.21$ ) and 2.5 times as high for having T4 levels tested (OR=2.51, 95\% CI=1.67-3.75). Odds of receiving PFT were also higher for pharmacist-managed than usual care patients (OR=5.89, 95\% CI=3.86-8.99). ORs for CXR did not reach statistical significance.

\section{Clinical Outcomes}

A higher proportion of patients in the pharmacist-managed group than in usual care were taking either lovastatin at more than $40 \mathrm{mg}$ per day or either simvastatin or atorvastatin at more than $20 \mathrm{mg}$ per day $(47.5 \%$ [n=86] vs. $36.2 \%$ [ $=764]$, respectively, $P=0.003$; Table 5). However, among those patients, a greater proportion was switched to either pravastatin or rosuvastatin $(14.0 \%[n=12]$ vs. $7.5 \%[n=57], P=0.037)$ or a lower dose of lovastatin, simvastatin, or atorvastatin $(9.3 \%$ $[\mathrm{n}=8]$ vs. $3.9 \%$ [ $\mathrm{n}=30], P=0.022)$ in the pharmacist-managed group than in usual care during the study period. There was no significant difference in the percentage of patients who discontinued statin therapy $(32.6 \%[\mathrm{n}=28]$ vs. $31.0 \%[\mathrm{n}=237]$, $P=0.771$ ).

Among those patients from the usual care group who used 1 of the 3 high-dose statins and amiodarone concomitantly, 6 patients $(0.8 \%$ of those using a high-dose statin, $0.3 \%$ of the usual care group overall) had an encounter with a primary diagnosis of rhabdomyolysis (Table 5). There were 5 hospitalizations and 3 ER visits associated with rhabdomyolysis. No patients in the pharmacist-managed group had an inpatient admission or ER visit with a primary diagnosis code for rhabdomyolysis.

The proportions of patients using amiodarone and digoxin concomitantly were similar in the 2 groups $(35.9 \%$ [n $=65]$ vs. $31.3 \%[\mathrm{n}=660], P=0.197$; data not shown). Among those, 6 in the pharmacist-managed group and 43 in usual care had serum digoxin levels more than 2 ng per $\mathrm{mL}$ ( $9.2 \%$ vs. $6.4 \%$ of concomitant amiodarone/digoxin users, respectively, $P=0.405$; data not shown). In the pharmacist-managed group, 1 patient was admitted to the hospital, and 1 went to the ER within 2 days of an elevated serum digoxin level, compared with 25 hospitalizations and $17 \mathrm{ER}$ visits in the usual care group (Table 5).

One patient in the pharmacist-managed group experienced an ALT exceeding $120 \mathrm{u}$ per L, which was associated with a hospitalization within 2 days following the elevated ALT, while 19 patients in usual care had elevated ALT with 7 hospitalizations and 1 ER visit. One patient in the pharmacist-managed program had abnormal thyroid function results, whereas 8 in usual care did, with 2 hospitalizations. Finally, in the pharmacist-managed program, there were no hospitalizations for interstitial lung disease after starting amiodarone treatment, compared with 5 in the usual care group.

\section{Estimate of Return on Investment}

There were 44 potentially avoidable hospitalizations in the usual care group at a cost of $\$ 372,345$. The cost for the $22 \mathrm{ER}$ visits was $\$ 9,922$, which yielded a total potential cost avoidance of $\$ 382,267$ (data not shown). The cost of the pharmacist-monitoring program for the study period was $\$ 10,425$ or approximately $\$ 58$ per patient, derived from the pharmacist's time of 10 hours per month multiplied by the pharmacist wage plus employee benefits rate of $\$ 86.88$ per hour. If the cost of the program is extrapolated to all other patients treated with amiodarone in our medical care program, the total cost of the pharmacist-managed program for the 2,111 usual care patients 
Evaluation of a Pharmacist-Managed Amiodarone Monitoring Program

TABLE 5 Clinical Outcomes Among Patients Enrolled in a PharmacistManaged Amiodarone Monitoring Program Versus Usual Care

\begin{tabular}{|c|c|c|c|c|c|c|c|c|}
\hline & \multicolumn{4}{|c|}{ Pharmacist-Managed $(\mathrm{n}=181)$} & \multicolumn{4}{|c|}{ Usual Care $(n=2,111)$} \\
\hline & $\begin{array}{l}\text { Patients } \\
\text { n (\%) }\end{array}$ & $\begin{array}{c}\text { Hospital } \\
\text { Admissions } \\
\mathbf{n}\end{array}$ & $\begin{array}{l}\text { Hospital } \\
\text { Days }^{\mathrm{a}}\end{array}$ & $\begin{array}{c}\text { ER Visits } \\
n\end{array}$ & $\begin{array}{l}\text { Patients } \\
\text { n (\%) }\end{array}$ & $\begin{array}{c}\text { Hospital } \\
\text { Admissions } \\
\mathbf{n}\end{array}$ & $\begin{array}{l}\text { Hospital } \\
\text { Days }^{a}\end{array}$ & $\begin{array}{c}\text { ER Visits } \\
n\end{array}$ \\
\hline$\overline{A L T} \geq 120 \mathrm{u}$ per $\mathrm{L}$ & $1 \quad(0.55)$ & 1 & $1 \quad(1.0)$ & 0 & $19 \quad(0.90)$ & 7 & $35 \quad(5.0)$ & 1 \\
\hline $\begin{array}{l}\text { TSH }<0.4 \text { or }>4.0 \text { uIU per } \mathrm{mL} \\
\text { and } \mathrm{T} 4<0.81 \mathrm{ng} \text { per } \mathrm{dL}\end{array}$ & $1 \quad(0.55)$ & 0 & 0 & 0 & $8 \quad(0.38)$ & 2 & $2(1.0)$ & 0 \\
\hline Serum digoxin $>2.0 \mathrm{ng}$ per $\mathrm{mL}$ & $6 \quad(3.31)$ & 1 & $5 \quad(5.0)$ & 1 & $43 \quad(2.04)$ & 25 & $175 \quad(7.0)$ & 17 \\
\hline Rhabdomyolysis ${ }^{\mathrm{b}, \mathrm{c}}$ & 0 & 0 & 0 & 0 & $6 \quad(0.28)$ & 5 & $12 \quad(2.4)$ & 3 \\
\hline \multirow[t]{3}{*}{ Interstitial lung disease } & 0 & 0 & 0 & 0 & $5 \quad(0.24)$ & 5 & $17 \quad(3.4)$ & 1 \\
\hline & \multicolumn{4}{|c|}{ Pharmacist-Managed $(n=181)$} & \multicolumn{4}{|c|}{ Usual Care $(n=2,111)$} \\
\hline & $\begin{array}{l}\text { Patients } \\
\text { n (\%) }\end{array}$ & $\begin{array}{c}\text { Discontinued } \\
\text { Statin } \\
\mathrm{n}(\%)\end{array}$ & $\begin{array}{c}\text { Switched to } \\
\text { Lower Dose } \\
\text { n (\%) }\end{array}$ & $\begin{array}{c}\text { Switched to } \\
\text { Pravastatin or } \\
\text { Rosuvastatin } \\
n(\%)\end{array}$ & $\begin{array}{l}\text { Patients } \\
\text { n (\%) }[P]^{\mathrm{d}}\end{array}$ & $\begin{array}{c}\text { Discontinued } \\
\text { Statin } \\
\mathbf{n}(\%)[P]\end{array}$ & $\begin{array}{c}\text { Switched to } \\
\text { Lower Dose } \\
\text { n (\%) }[P]\end{array}$ & $\begin{array}{l}\text { Switched to } \\
\text { Pravastatin or } \\
\text { Rosuvastatin } \\
\text { n }(\%)[P]\end{array}$ \\
\hline High-dose statinc & $86(47.5)$ & $28(32.6)$ & $\begin{array}{ll}8 & (9.3)\end{array}$ & $12(14.0)$ & $\begin{array}{c}764(36.2) \\
{[0.003]}\end{array}$ & $\begin{array}{c}237(31.0) \\
{[0.771]}\end{array}$ & $\begin{array}{c}30 \\
{[0.022]}\end{array}$ & $\begin{array}{c}57 \quad(7.5) \\
{[0.037]}\end{array}$ \\
\hline
\end{tabular}

aTotal hospital days (mean number of days).

${ }^{b}$ Rhabdomyolysis determined by ICD-9-CM principal diagnosis code 728.88 among patients with concomitant amiodarone and high-dose statin use at months 1-12.

'High-dose statin is defined as lovastatin more than $40 \mathrm{mg}$ per day, simvastatin more than $20 \mathrm{mg}$ per day, or atorvastatin more than $20 \mathrm{mg}$ per day.

${ }^{d}$ Wald chi-square statistical test for pharmacist-managed versus usual care.

$A L T=$ alanine aminotransferase; $d L=$ deciliter $; E R=$ emergency room; ICD-9-CM=International Classification of Diseases, Ninth Revision, Clinical Modification; $L=$ liter; $m g=$ milligrams; $m L=$ milliliter; $n g=$ nanogram; $T 4=$ thyroxine; $T S H=$ thyroid-stimulating hormone; $u=$ units; uIU = micro international units

would be approximately $\$ 121,587$. Assuming that all hospitalizations and ER visits in the usual care group were avoidable and based on the ROI calculation of the difference between the costs avoided and the cost of the program, divided by the cost of the program, we estimate that for every dollar spent on the program, $\$ 2.14$ ([\$382,267-\$121,587]/ $\$ 121,587)$ could be saved through reduced hospitalizations and ER visits related to adverse drug events among patients treated with amiodarone.

\section{Discussion}

We found that a pharmacist-managed amiodarone program was associated with an increase in the percentage of patients who received laboratory monitoring and PFTs compared with usual care. The trends in monitoring rates for ALT were similar to those in the study by Johnson et al. of the pharmacist-managed AMS in KP Colorado, which found significantly improved ALT monitoring post-AMS compared with pre-AMS (84\% vs. $69 \%$ at 1 year). ${ }^{14}$ However, Johnson et al. did not find a significant improvement in TSH monitoring (68\% vs. 64\% at 1 year) and suggested that monitoring was higher for ALT because it was routinely performed for patients prescribed statins. The Johnson et al. study also found that adherence to CXR monitoring rates in the first year of amiodarone therapy were significantly lower post-AMS than pre-AMS (53\% vs. $71 \%$ ). The CXR rate during 1 year of post-index follow-up in the present study (56.9\% in pharmacist-managed care) did not significantly differ from $50.0 \%$ in the usual care group and was similar to the AMS rate (53\%) in the study by Johnson et al. CXR requires authorization from a physician, as opposed to routine blood tests, which could be initiated by a pharmacist. In addition, appointments must be booked for CXRs, whereas the patient can get a laboratory test done at any time.

For PFT, the study by Bickford and Spencer found that 24\% of patients had received these tests at baseline, and the authors suggested room for improvement. ${ }^{11}$ The baseline PFT monitoring rates in the present study $(6.6 \%$ in the pharmacist-managed group and 3.6\% in usual care) were substantially lower than that reported by Bickford and Spencer, and during the first 12 months post-index, the PFT monitoring rate in the present study was only slightly more than $50 \%$ in the pharmacistmanaged group. Thus, there is also room for improvement at our institution. PFTs also require booking an appointment, and the procedure is available only at the main medical center, unlike CXRs, which can be obtained at clinics outside of the main medical center.

We found very few cases of liver-related adverse events. This finding is consistent with clinical trial data where the incidence of liver toxicity has been reported as $0.6 \%{ }^{4}$ Johnson et al. used ICD-9-CM codes and manual chart review to identify liverrelated adverse event rates of $0.8 \%$ in the AMS group and $1.7 \%$ prior to AMS implementation. Thyroid-related events (also defined using ICD-9-CM codes for thyroid disease and confirmed through manual chart review) occurred in 3.9\% of the AMS group and $6 \%$ of patients prior to AMS implementation. ${ }^{14}$ We found very small numbers of patients with abnormal TSH and T4 lab results who were hospitalized or had an ER visit. 
The difference may be one of measurement: the Johnson et al. study was based on any hospitalizations or ER visits related to thyroid toxicity, which may have captured more patients than did the present study, which identified patients based on abnormal laboratory test results first and then looked for hospitalizations and ER visits within the subsequent 2-day period. Alternatively, the higher rates of thyroid monitoring in the present study may have led to fewer adverse events. The incidence of pulmonary toxicity has been reported at $1.1 \%$ from clinical trial data. ${ }^{4}$ Johnson et al. found pulmonary adverse event rates of $0.8 \%$ in the AMS group and $1.9 \%$ pre-AMS. We found no pulmonary adverse events in the pharmacist-managed group and $0.2 \%$ in usual care.

There appears to be a continuing need for health plans to monitor patient exposure to the combination of amiodarone and high-dose statins. Snider et al. reported that the most common adverse events that were identified by the clinical pharmacists in their antiarrhythmic monitoring clinic were clinically significant drug interactions, the most frequent being amiodarone and high-dose simvastatin. ${ }^{17}$ In the study by Karimi et al. (2010) of a safety initiative for patients on concomitant amiodarone and simvastatin, 92 patients, representing $0.52 \%$ of simvastatin users, were using these medications concomitantly for an average of 43 months at a VA medical center. ${ }^{21}$ Karimi et al. did not assess adverse events, but a review of 30 clinical trials showed the rates of rhabdomyolysis for statins versus placebo to be $0.016 \%$ and $0.012 \%$, respectively. ${ }^{22}$ An FDA safety alert issued in August 2008 reported 52 cases of rhabdomyolysis in patients receiving concomitant amiodarone and simvastatin in doses greater than $20 \mathrm{mg}$ per day. We found 6 cases in the usual care group ( $0.79 \%$ of those on high-dose statins). Although some patients in both groups were switched to a safer statin (pravastatin or rosuvastatin) or a lower dose, about 30\% discontinued statin use altogether. One explanation could be that because pravastatin and rosuvastatin are not formulary agents (the only statin formulary drugs are simvastatin and lovastatin), patients may have preferred to stop using a statin rather than pay higher out-of-pocket costs. Additional research on patient behavior regarding switching to safer alternatives among nonformulary medications would be beneficial.

The study by Graham et al. (2004) conducted at the VA found that $41 \%$ of patients enrolled in their pharmacist-managed AMC and 21\% in the control group were on concomitant digoxin therapy. ${ }^{15}$ The mean (SD) digoxin drug concentration was $0.87(0.50)$, and the authors did not report any adverse effects related to digoxin use. The proportion of patients in the present study on concomitant digoxin was within the range reported in the study by Graham et al. However, $1 \%$ of patients in the pharmacist-managed group and $2 \%$ in usual care had elevated digoxin concentrations and were either hospitalized or were seen in the ER. This was the most common adverse event in the present study, which suggests a need for increased vigilance of patients on concomitant amiodarone and digoxin.
An economic analysis by Perez et al. (2008) of clinical pharmacy services from 2001-2005 found that the median ROI was $\$ 4.81$ (range \$1.02-\$34.61). ${ }^{23}$ These services were mostly hospital based, which may account for the higher ROI compared with the present study. However, 2 ambulatory care clinics, which reported rates of $\$ 1.02$ and $\$ 2.89$, were included in the analysis by Perez et al.

\section{Limitations}

Foremost among the study limitations is that patients were not randomized to the pharmacist-managed group versus usual care; therefore, it is not possible to say that the pharmacistmanaged care was responsible for the higher rates of laboratory monitoring. It is possible that the results were attributable to systematic bias introduced by selection into usual care versus pharmacist-managed amiodarone care or that the 2 groups differed in other ways, such as socioeconomic status and ease of transportation, which may have affected monitoring or the patients' ability to complete their recommended tests. We used covariates to adjust for age, gender, race, new versus continuing use, and DxCG, but we cannot rule out the potential for selection bias. Second, laboratory tests and PFTs may have been ordered for a reason other than monitoring for amiodarone treatment and therefore might overestimate the prevalence of recommended testing. Third, this was a retrospective database analysis, and the data were not verified by a more detailed medical chart (EMR) review. Therefore, there could be potential misclassification of some measures, especially the drugdrug interactions. For example, some drugs may have been purchased but not taken, or the patients may not have taken them simultaneously. Patients may also have been told verbally to take a reduced dose or stop taking a medication, and this instruction would not be captured in the database. Fourth, the sample size of the pharmacist-managed group may have been too small to adequately detect outcomes of low frequency, such as interstitial lung disease and rhabdomyolysis. Fifth, the ROI analysis assumed that all usual care group hospitalizations and ER visits were avoidable, but a small number of these events occurred in the pharmacist-managed group. Finally, we did not assess patient or physician satisfaction. However, we have received positive feedback from physicians, which has played a role in expanding the program to the other medical centers in the KPSC region.

\section{Conclusions}

This study suggests that pharmacist management of patients treated with amiodarone can improve rates of monitoring of recommended laboratory tests and PFTs. Increased monitoring may potentially reduce hospitalizations and ER visits associated with failure to adjust drug regimens and dosages for amiodarone and drugs that interact with amiodarone, specifically digoxin and high-dose statins. 


\section{Authors}

MICHELE M. SPENCE, PhD, is Pharmacy Outcomes Project Manager, Pharmacy Outcomes Research Group, Kaiser Permanente, Downey, California. JENNIFER K. POLZIN, PharmD, is Clinical Operations Manager, Ambulatory Care Pharmacy; CALVIN L. WEISBERGER, MD, is Staff Cardiologist, Internal Medicine; JOHN P. MARTIN, MD, is Staff Internist, Internal Medicine; JAY P. RHO, PharmD, is Area Pharmacy Director, Pharmacy Operations; and GISELLE H. WILLICK, PharmD, is Clinical Operations Manager, Kaiser Permanente, Los Angeles, California.

AUTHOR CORRESPONDENCE: Michele M. Spence, PhD, Kaiser Permanente, Pharmacy Outcomes Research Group, 12254 Bellflower Blvd., Downey, CA 90242. Tel.: 562.658.3528;

E-mail: michele.m.spence@kp.org.

\section{DISCLOSURES}

There was no external funding for this research, and the authors report no financial or other potential conflicts of interest related to the subject of this manuscript.

Concept and design were performed by Polzin and Spence. Data were collected by Polzin, Spence, Weisberger, and Willick and interpreted by Spence with the assistance of Martin and Polzin. The manuscript was written by Spence and revised by Martin, Polzin, Rho, and Spence.

\section{REFERENCES}

1. Cordarone (amiodarone HCL) tablets. Wyeth Pharmaceuticals. December 2010. Available at: http://labeling.pfizer.com/showlabeling.aspx?id=93. Accessed August 23, 2011.

2. Zimetbaum P, Ho KKL, Olshansky B, et al.; FRACTAL Investigators. Variation in the utilization of antiarrhythmic drugs in patients with newonset atrial fibrillation. Am J Cardiol. 2003;91(1):81-83.

3. Siddoway LA. Amiodarone: guidelines for use and monitoring. Am Fam Physician. 2003;68(11):2189-96. Available at: http://www.aafp.org/ afp/2003/1201/p2189.html. Accessed August 23, 2011.

4. No authors listed. Effect of prophylactic amiodarone on mortality after acute myocardial infarction and in congestive heart failure: meta-analysis of individual data from 6500 patients in randomised trials. Lancet. 1997;350(9089): 1417-29.

5. Hilleman D, Miller MA, Parker R, Doering P, Pieper JA. Optimal management of amiodarone therapy: efficacy and side effects. Pharmacotherapy. 1998;18(6 Pt 2):138S-145S.

6. Vorperian VR, Havighurst TC, Miller S, January CT. Adverse effects of low dose amiodarone: a meta-analysis. J Am Coll Cardiol. 1997;30(3):791-98.

7. Dusman R, Stanton MS, Miles WM, et al. Clinical features of amiodaroneinduced pulmonary toxicity. Circulation. 1990;82(1):51-59.

8. U.S. Food and Drug Administration. Interaction between amiodarone (marketed as CORDARONE and PACERONE) and simvastation (marked as ZOCOR and generics) or simvastation-combination products (marketed as VYTORIN and SIMCOR). Amiodarone potentiates the risk for simvastation-associated rhabdomyolysis. Postmarketing Reviews, vol. 1, no. 1. Summer 2008. Available at: http://www.fda.gov/Drugs/DrugSafety/ DrugSafetyNewsletter/ucm120613.htm\#amiodarone_simvastatin. Accessed August 23, 2011.
9. Goldschlager N, Epstein AE, Naccarelli GV, et al.; the Practice Guidelines Subcommittee and the North American Society of Pacing and Electrophysiology. A practical guide for clinicians who treat patients with amiodarone: 2007. Heart Rhythm. 2007;4(9):1250-59.

10. Raebel MA, Carroll NM, Simon SR, et al. Liver and thyroid monitoring in ambulatory patients prescribed amiodarone in $10 \mathrm{HMOs.J}$ Manag Care Pharm. 2006;12(8):656-64. Available at: http://amcp.org/data/jmcp/656-664. pdf.

11. Bickford CL, Spencer, AP. Adherence to the NASPE guideline for amiodarone monitoring at a medical university. J Manag Care Pharm. 2006;12(3):254-59. Available at: http://www.amcp.org/WorkArea/ DownloadAsset.aspx?id=7351.

12. Tjia J, Field TS, Garber LD, et al. Development and pilot testing of guidelines to monitor high-risk medications in the ambulatory setting. Am J Manag Care. 2010;16(7):489-96.

13. Johnson SG. Improving cost-effectiveness of and outcomes from drug therapy in patients with atrial fibrillation in managed care: role of the pharmacist. J Manag Care Pharm. 2009;15(6 Suppl B):19S-25S. Available at: http://www.amcp.org/data/jmcp/1003.19-25.pdf.

14. Johnson SG, Canty K, Billups S, Schimmer J. Adherence to amiodarone monitoring recommendations before and after implementation of a centralized pharmacy service: a cohort study. J Pharm Pract. 2010;23(6):536-39.

15. Graham MR, Wright MA, Manley HJ. Effectiveness of an amiodarone protocol and management clinic in improving adherence to amiodarone monitoring guidelines. J Pharm Technol. 2004;20:5-10. Available at: http:// jpharmtechnol.com/abstracts/volume20/January-February/5.html. Accessed August 23, 2011.

16. Raebel MA, Lyons EE, Chester EA, et al. Improving laboratory monitoring at initiation of drug therapy in ambulatory care. Arch Intern Med. 2005;165(20):2395-401. Available at: http://archinte.ama-assn.org/cgi/content/full/165/20/2395. Accessed August 23, 2011.

17. Snider M, Kalbfleisch S, Carnes CA. Initial experience with antiarrhythmic medication monitoring by clinical pharmacists in an outpatient setting: a retrospective review. Clin Ther. 2009;31(6):1209-18.

18. Sanoski CA, Schoen MD, Gonzalez RC et al. Rationale, development, and clinical outcomes of a multidisciplinary amiodarone clinic. Pharmacotherapy. 1998;18(6 Pt 2):146S-151S.

19. Solberg LI, Hurley JS, Roberts MH, et al. Measuring patient safety in ambulatory care: potential for identifying medical group drug-drug interaction rates using claims data. Am J Manag Care. 2004;10(11 Pt 1):753-59.

20. Ash AS, Ellis RP, Pope GC, et al. Using diagnoses to describe populations and predict costs. Health Care Financ Rev. 2000;21(3):7-28. Available at: http://www.cms.gov/HealthCareFinancingReview/Downloads/00springpg7. pdf. Accessed August 23, 2011.

21. Karimi S, Hough A, Beckey C, Parra D. Results of a safety initiative for patients on concomitant amiodarone and simvastatin therapy in a Veterans Affairs medical center. J Manag Care Pharm. 2010;16(7):472-81. Available at: http://www.amcp.org/data/jmcp/472-481.pdf.

22. Thompson PD, Clarkson P, Karas RH. Statin-associated myopathy. JAMA. 2003;289(13):1681-90.

23. Perez A, Doloresco F, Hoffman JM, et al. Economic evaluations of clinical pharmacy services: 2001-2005. Pharmacotherapy. 2009;29(1):128. 